\title{
Toma de decisiones en escenarios complejos
}

\section{Decision making in complex scenarios}

\section{Prise de décisions en situations complexes}

\section{A tomada de decisão em cenários complexos}

\section{Jorge Augusto Pérez Alcázar* Johan Manuel Redondo**}

Fecha de recepción: 9 de febrero

Fecha de aprobación: $\mathbf{8}$ de marzo

Pp. 121-142

* Magíster en Educación con énfasis en Análisis Matemático, Universidad Pedagógica Nacional.; especialista en Matemática Aplicada, Universidad Sergio Arboleda; especialista en Sistemas Dinámicos, Universidad Surcolombiana; licenciado en Matemáticas, Universidad Pedagógica Nacional y profesor asociado tiempo completo en la Facultad de Ingeniería Universidad EAN.

** PhD. Doctor en Ingeniería Automática, Universidad Nacional de Colombia; magíster en Gestión y Evaluación Ambiental, magíster en Docencia e Investigación Universitaria, especialista en Matemática Aplicada, Universidad Sergio Arboleda; ingeniero ambiental, Corporación Universitaria De Ciencia Y Desarrollo - UNICIENCIA y catedrático de la Universidad EAN. 


\section{RESUMEN}

En este trabajo se presenta una metodología novedosa para la toma de decisiones basada en la dinámica del sistema y los sistemas dinámicos. Aquí se muestra cómo el diagrama de bifurcaciones es en realidad un mapa de todos los escenarios posibles. Esta herramienta es muy importante para los gerentes y los administradores públicos, porque el ejercicio de la toma de decisiones se torna más sencillo cuando la comprensión del problema se ve mejorada. En este documento mostraremos algunos alcances de esta metodología usando como ejemplo un modelo para la creación de empresas en una zona industrial.

\section{Palabras Clave}

Sistema dinámicos, bifurcaciones, decisión máquina, complex escenarios.

\section{Abstract}

This paper presents an innovative decision-making methodology, based on the dynamics of the system and the systems dynamics. This document shows how the bifurcation diagram is in fact, a map of all the possible scenarios. This tool is very important for managers and public administrators, because the exercise of decision making becomes easier when the understanding of the problem is improved. This document will show some achievements of this methodology by using a model for entreprise creation in an industrial area as an example.

\section{KEYWORDS}

Dynamic system, bifurcations, decision machine, complex scenarios. 


\section{RÉSUMÉ}

Nous présenterons dans ce travail une nouvelle $\neg$ méthodologie pour la prise de décisions basée sur la dynamique du système et les systèmes dynamiques. Nous montrerons de quelle manière le diagramme de bifurcations se trouve être en réalité une carte de tous les scénarios possibles. Cet outil est en effet très important pour les gérants et gestionnaires publics car l'exercice de la prise de décisions devient plus simple lorsque la compréhension du problème s'améliore. Nous montrerons dans ce document la portée de cette méthodologie en prenant comme exemple un modèle de création d'entreprise en zone industrielle.

\section{MOTS CLEFS}

Système dynamiques, bifurcations, décision, machine, scénarios complexes.

\section{Resumo}

Este artigo apresenta uma nova metodologia para a tomada de decisão com base na dinâmica de sistema e sistemas dinâmicos. Aqui se mostra como o diagrama de bifurcação é na verdade um mapa de todos os cenários possíveis. Esta ferramenta é muito importante para os gestores e administradores públicos, porque o exercício de tomada de decisões torna-se mais fácil quando a compreensão do problema é melhorada. Neste documento mostraremos alguns alcances dessa metodologia utilizando como exemplo um modelo para a criação de empresas em uma zona industrial.

\section{Palavras-chave}

Sistema dinâmico, bifurcações, máquina de decisão, cenários mais complexos. 


\section{Introducción}

a toma de decisiones es el proceso más central y persistente de las actividades del ser humano, intrínseca a su biología y realizada tanto consciente como inconscientemente. El mejoramiento continuo de este proceso es el elemento crucial para su sobrevivencia y desarrollo como especie (Garuti, y Escudey, 2005).

Dado que el futuro es incierto es necesario prepararse para múltiples posibles futuros y no solo para el que se espera que suceda (Bishop, Hines, y Collins, 2007). Pero ¿cómo es posible hacer la construcción de escenarios si estos describen situaciones futuras junto con la progresión de eventos desde la situación base hasta la situación futura? (Godet, 2007).

En este documento se pretende formular una alternativa a este cuestionamiento, basándose en la teoría de los sistemas dinámicos no lineales -teoría Matemática- partiendo de la metodología conocida como dinámica de sistemas -metodología de la ingeniería- aplicable, en principio, a la empresa pero extensible a diferentes tipos de sistemas.

Para ello, hay que empezar por decir que «la empresa es un sistema técnico-social abierto. Su función básica es crear bienes y servicios que contribuyan a elevar el nivel de vida de la humanidad» (Fernández, 2005).

Lo anterior, configura el primer marco de lo que se busca en esta investigación: constituir a la empresa en un sistema. Lo deseable es que este sistema alcance «los objetivos marcados (eficacia), pero utilizando bien los recursos disponibles (eficiencia)» (Fernández, 2005). Aquí emerge de forma natural la gestión, pues es esta quien colabora en el logro de tales fines. 
Ahora, la empresa no es un sistema estático, inmutable, «la empresa es también un sistema dinámico, con un ciclo biológico de nacimiento, desarrollo, madurez y declive» (Fernández, 2005). Este sistema dinámico es adicionalmente un sistema abierto en el sentido que hace intercambios de información, de materia y de energía con su entorno. Esto hace que la empresa experimente fenómenos de emergencia y constreñimiento, de realimentación y de recursividad, los cuales son síntomas de los sistemas complejos (Morin, 2008) que deben ser abordados en el ejercicio de la comprensión desde el uso de las denominadas ciencias de la complejidad.

Para descubrir el valor de estas ciencias se de volver al concepto de gestión ya mencionado. La gestión puede interpretarse como el conjunto de acciones que garantizan el cumplimiento de los objetivos de la empresa haciendo buen manejo de los recursos de esta (Merli, 1997).

Merli (1997) señala que la gestión es eficaz cuando existe una mayor capacidad para gestionar los breakthroughs,Breakthroughs: mejoras drásticas de los resultados (rupturas, conquistas)-, es decir, cuando se desarrolla la habilidad para:

- Elegir los objetivos correctos: «las mejoras que se logren deben tener efecto en las palancas correctas».

- Planificar las acciones necesarias para alcanzar los objetivos. Es decir, elaborar planes eficaces y coherentes.

- Convertir los objetivos en resultados.

- Convertir las mejoras logradas en resultados consolidados e irreversibles. En otras palabras, consolidar los resultados alcanzados. 
El problema de esta gestión eficaz, está en la elección de los objetivos correctos ¿cómo saber que se está aplicando esfuerzo para el movimiento de las palancas correctas?

Senge (s.f) cita lo que se conoce como el juego de la cerveza, en el que tres niveles distintos del mercado toman sus decisiones sobre los objetivos incorrectos haciendo que el mercado entre en crisis. Al momento de preguntársele a los actores sobre sus comportamientos, todos estaban seguros de haber identificado bien sus objetivos y las acciones a tomar para satisfacerlos, luego, no se explicaban cómo sucedió, cómo haciendo todos las cosas bien, terminaron perdiendo.

Esto lleva al punto central de este documento, pues la gestión depende sensiblemente de los objetivos planteados, los cuales, a su vez, dependen de la comprensión que se tiene del sistema: la empresa. Esta comprensión no puede hacerse de forma aislada, es decir, ubicando a la empresa fuera de sus contextos, porque como ya se ha dicho, la empresa es un sistema abierto. Tampoco debe interpretarse a la empresa como la unidad mínima de comprensión, porque esta es, al mismo tiempo, un subsistema de un sistema comercial más grande, y un sistema de sistemas, en el sentido que muchas de sus operaciones internas están configuradas en el marco de sistemas que los sustentan.

Desde este punto de vista, la comprensión de nuestro sistema es el insumo más importante de la organización y no debería escatimarse en esfuerzos para de forma continua mejorar en este aspecto, tanto desde el punto de vista de la acumulación de información como del mejoramiento de las metodologías que en este sentido se aplique.

Al respecto, en este documento se presentan los diagramas de bifurcaciones de la teoría de los Sistemas Dinámicos No Lineales, como la posibilidad de obtener mapas de todos los escenarios posibles de un sistema y, a partir de ellos, identificar 
prospectivamente los posibles escenarios a los que tiene que enfrentarse la organización, lo cual le permite establecer los objetivos que apalancarán exitosamente a la empresa.

Esta posibilidad ubica en otro marco el diseño de políticas y el ejercicio gerencial, dándole la posibilidad de diseñar estrategias y de tomar decisiones fuera del contexto del ensayo y error, además de corresponderse al estilo de pensamiento que se pretende buscar actualmente: el pensamiento no lineal.

\section{Modelamiento y análisis de sistemas}

Antes de materializar la propuesta de este documento, se presenta el problema BSNSS3 desarrollado por Alfeld \& Graham (1976) y Aracil y Gordillo (1997), en donde se modela el crecimiento económico de un área industrial utilizando la metodología de la dinámica de sistemas.

Esta metodología, consiste de tres etapas: conceptualización, formulación y evaluación (Aracil y Gordillo, 1997). En la etapa de conceptualización se obtiene como producto principal un diagrama causal en el que se visualiza el problema de forma sistémica, en donde las partes del sistema se denominan atributos y las relaciones son de tipo causal. En la etapa de formalización se construye un diagrama que busca formalizar el diagrama causal, clasificando los atributos del sistema en variables de estado, razones de cambio, variables auxiliares y parámetros; el diagrama se denomina diagrama de Forrester o diagrama de niveles y flujos. A partir del diagrama de Forrester se puede establecer un conjunto de ecuaciones que, al componerse, constituyen un sistema de ecuaciones 
diferenciales ordinarias de primer orden, las cuales representan la dinámica del sistema.

EI mencionado problema BSNSS3 es presentado como sigue:

Se supone un área de superficie limitada en la que se produce un proceso de establecimiento de empresas. Como consecuencia de ello el número de las empresas crece. Este crecimiento viene afectado por el grado de ocupación del área, de modo que cuando hay todavía pocas empresas es fácil el establecimiento de nuevas, mientras que cuando el área está prácticamente ocupada se producen fenómenos de saturación que tienden a limitar el crecimiento. Por otra parte, se supone que se produce un flujo, relativamente pequeño de desaparición de empresas, por obsolescencia, quiebra o razones análogas (Aracil y Gordillo, 1997).

\subsection{Dinámica de sistemas}

De acuerdo con la descripción anterior, se construye el diagrama causal, que como se ve es un conjunto de grafos dirigidos bi-valuados por medio de los cuales queda organizada causalmente la información del problema (Figura 1).

Figura 1. Diagrama causal

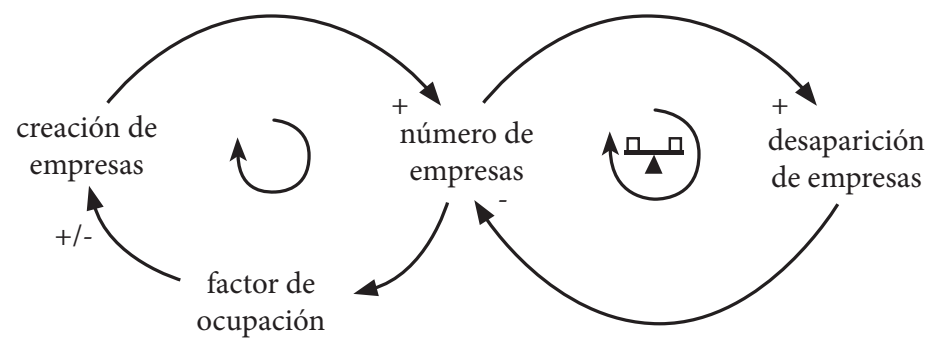

Fuente. Aracil y Gordillo, (1997). 
Como se puede observar, el número de empresas es consecuencia de la creación de empresas y causa de la desaparición de empresas. A mayor desaparición de empresas, menor será el número de empresas en el área industrial. Ahora, el mayor número de empresas hace que el factor de ocupación del área industrial sea mayor, y este factor de ocupación, a su vez, determina la creación de empresas.

A continuación (Figura 2), se representa el efecto que ejerce el factor de ocupación del área industrial sobre la creación de empresas. De acuerdo ello, para valores pequeños del factor de ocupación FO, el multiplicador MES crece conjuntamente con $F O$, sin embargo, a partir de un valor especifico de $F O$, el multiplicador decrece con el aumento del factor de ocupación hasta anularse cuando toda el área esta edificada. Es importante anotar, además, que la función $f$ introduce una no linealidad en el sistema. La función $f$ es lo que se conoce como una función tabla.

Figura 2. Multiplicador de empresas en función de la superficie edificada

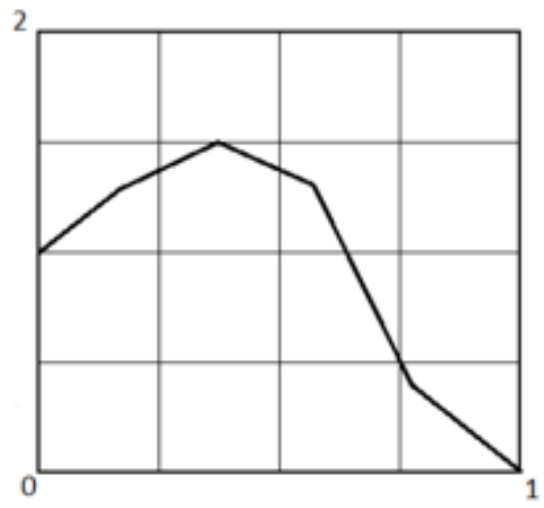

Nota. En la gráfica vemos que MES=f (FO).

Fuente. Aracil y Gordillo, (1997). 
De esta forma, se conforman dos bucles de realimentación, uno negativo que hace tender el sistema a un límite, y el otro cuyo signo no es posible determinar, debido a que incrementos del factor de ocupación, en valores pequeños de este, genera incrementos en la creación de empresas; pero incrementos del factor de ocupación para valores altos del mismo, provocan disminución en la creación de empresas, tal como se visualiza en la función tabla (Figura 2). Se debe saber que el factor de ocupación no se relaciona directamente con la creación de empresas porque esto no es correcto en el contexto real del problema, sino que es relacionado con la variable MES.

A partir del diagrama causal se avanza en la construcción del diagrama de niveles y flujos (Figura 3). En este se identifica una única variable de estado del sistema, el número de empresas NE. También, se identifican los flujos o razones de cambio del sistema: la creación de empresas CE y la desaparición de empresas $D E$. Finalmente, se identifican las variables auxiliares, que para este caso de estudio son el factor de ocupación $F O$ y el multiplicador de empresas en función de la superficie edificada MES.

Figura 3. Diagrama de niveles y flujos

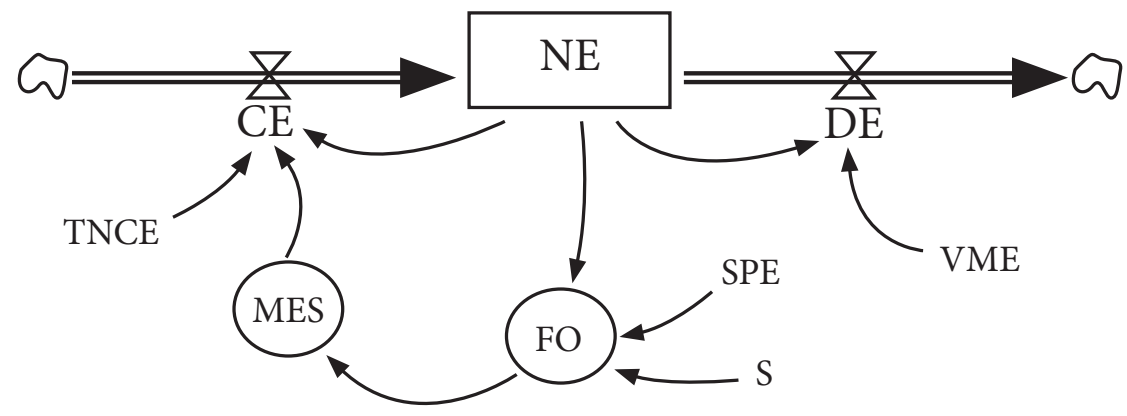

Fuente. Aracil y Gordillo, (1997). 
De acuerdo con la representación anterior, el parámetro TNCE es la tasa normal de creación de empresas, $S$ es la superficie edificable, SPE es la superficie media por empresa y $V M E$ es la vida media por empresa. Estos valores son los parámetros del modelo.

Desde este punto se construyen un conjunto de ecuaciones que describirán la evolución temporal del sistema, las cuales se presentan a continuación:

$$
\begin{aligned}
& \frac{d}{d t} N E=C E-D E \\
& C E=T N C E \cdot N E \cdot M E S \\
& D E=\frac{N E}{V M E} \\
& F O=\frac{S P E}{S} N E \\
& M E S=f(F O)
\end{aligned}
$$

La composición de estas ecuaciones da lugar a la ecuación diferencial que se presenta a así:

$$
\frac{d}{d t} N E=T N C E \cdot N E \cdot f\left(\frac{S P E}{S}\right) N E-\frac{N E}{V M E}
$$

La ecuación (6) es lo que se denomina el modelo determinístico del sistema. Simulaciones del sistema se realizaron en el software especializado de distribución gratuita Vensim PLE, obteniendo como resultados los mostrados a continuación (Figura 4). 
Figura 4. Simulaciones del sistema
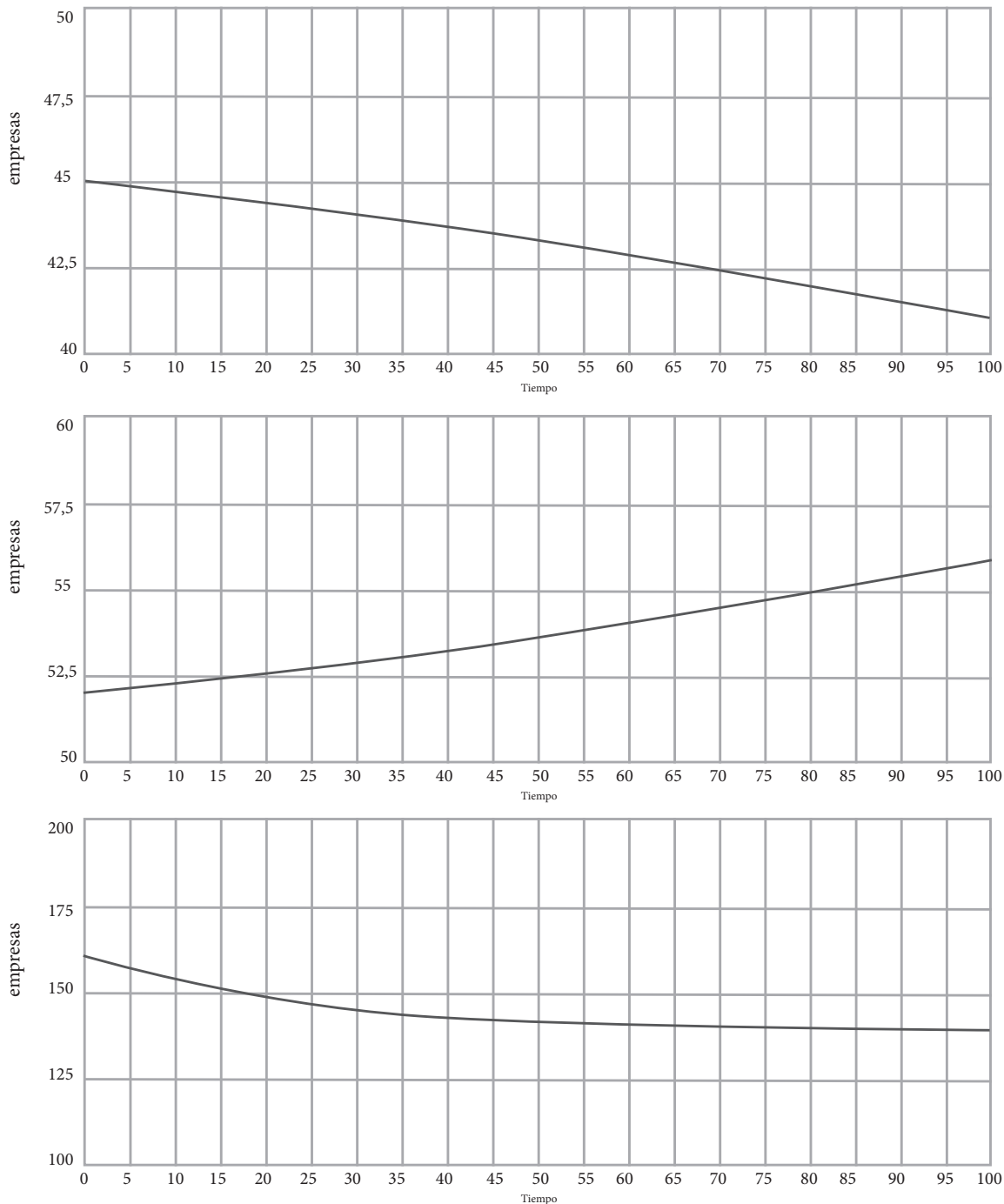

Nota. Para cada simulación se utilizaron condiciones iniciales distintas. En la primera gráfica el número de empresas inicial fue 45 , en la segunda 52 y en la tercera 160.

Fuente. Elaboración propia de los autores. 
A partir de las simulaciones surgen las siguientes preguntas: ¿Qué sucede entre 45 y 52 empresas que el sistema cambia su comportamiento y pasa de decreciente a creciente? y ¿por qué cuando se toma el valor 160 empresas, el sistema vuelve a decrecer? Es en este momento cuando la toma de decisiones necesita un criterio adicional, pues como se ve, es posible simular el sistema y, sin embargo, la comprensión no se experimenta completa como para la toma de una decisión segura.

\subsection{Sistemas dinámicos}

El criterio adicional que permite contestar las citadas preguntas se puede obtener de la teoría de los Sistemas Dinámicos No Lineales. Los sistemas dinámicos tienen como principal objetivo dar una caracterización completa de la estructura de las trayectorias del sistema y, si el sistema depende de parámetros, entonces caracterizar los cambios en la estructura de las trayectorias por la variación de los valores de los parámetros (Wiggins, 1990). Esto puede verse muy matemático, pero como se verá más adelante, esta es la teoría que se está buscando para tener comprensión completa del problema.

Lo primero que se realizará para simplificar la notación, son los siguientes cambios: $x:=N E, b:=1 / V M E, a:=$ TNCE y $k:=$ $S P E / S$, con lo que se obtiene la ecuación que sigue:

$$
x:=a \cdot x \cdot f(k x)-b \cdot x
$$

Para caracterizar completamente la estructura de las trayectorias, de acuerdo con la teoría de los Sistemas Dinámicos No Lineales, lo primero que se realiza es la identificación de los puntos de equilibrio del sistema, es decir, aquellos valores de la variable de estado para los que el sistema no cambia - es importante recordar que la variable de estado es el número de empresas-. Así, si por ejemplo, 32 empresas fuera un valor de equilibrio, entonces en el tiempo este valor será fijo, ni crecerá 
ni decrecerá. Esto es útil porque la dinámica de cualquier sistema unidimensional, como el nuestro, tiene tres tipos de comportamiento: o se aleja de algo o se acerca a algo o es ese algo, y lo que se ha denominado aquí como algo no es otra cosa que el equilibrio del sistema.

Los equilibrios del sistema se establecen calculando cuándo el sistema no cambia, es decir, cuándo $x=0$. Igualando la ecuación (7) a cero, se ecuentra:

$$
\begin{aligned}
& x=0 \\
& a \cdot x \cdot f(k x)-b \cdot x=0 \\
& x=0 \quad y \\
& a \cdot f(k x)-b=0 \\
& f(k x)=b / a=p \\
& k x=f^{-1}(p) \\
& x=1 / k f^{-1}(p)
\end{aligned}
$$

De donde se concluye que los equilibrios de este sistema son $x=0$ y $x=(1 / k) f^{-1}(p)$. Dado que la función $f$ es la función tabla y esta función no es lineal, la expresión $f^{1}$ no es la inversa sino el mapeo inverso a través del cual se calculan los valores de equilibrio. Hay que tener en cuenta que el mapeo $f^{-1}$ actúa sobre el valor de $p=b / a$, que corresponde con los valores que pueden ser obtenidos del eje vertical de la función tabla; luego, al mapear la inversa, se pueden encontrar uno, dos o ningún punto de equilibrio adicional al nulo.

El siguiente paso dentro de la metodología de la teoría de los Sistemas Dinámicos No Lineales es el establecimiento de la estabilidad del punto de equilibrio, es decir, si el punto de equilibrio es atractivo -el sistema tiende al punto de equilibrio- 
o repulsivo -el sistema se aleja del punto de equilibrio-. Este ejercicio se realiza a partir de la componente lineal del campo vectorial definido por la ecuación (7), que se denomina la jacobiana del sistema J, la cual, para el caso uno dimensional, se define como sigue:

$$
J(x)=\frac{\partial}{\partial x}(\dot{x})
$$

El objetivo es calcular la jacobiana sobre los puntos de equilibrio. Los valores obtenidos se denominan autovalores de la jacobiana. Si el total de los autovalores es negativo, se dice que el punto de equilibrio es atractivo, en caso contrario, al menos uno positivo, entonces el punto de equilibrio es repulsivo.

El cálculo de la jacobiana se muestra a continuación:

$$
\begin{aligned}
& J(x)=\frac{\partial}{\partial x}(\dot{x})=\frac{\partial}{\partial x}(a \cdot x \cdot f(k x)-b \cdot x) \\
& J(x)=\frac{\partial}{\partial x}(a \cdot x) \cdot f(k x)+a \cdot x \cdot \frac{\partial}{\partial x} f(k x)-\frac{\partial}{\partial x}(b \cdot x) \\
& J(x)=a \cdot f(k x)+a \cdot x \cdot f^{\prime}(k x) \cdot k-b
\end{aligned}
$$

Que sobre el punto de equilibrio nulo conduce al resultado:

$$
\begin{aligned}
& J(0)=a \cdot f(0)+a \cdot 0 \cdot f^{\prime}(0) \cdot k-b \\
& J(0)=a \cdot f(0)-b
\end{aligned}
$$

Pero por definición $f(0)=1$, con lo que:

$$
J(0)=a-b
$$


De donde se definen dos casos. El primer caso es cuando la diferencia $a-b>0$, es decir, $a>b$ o de la ecuación (11), $0<p<1$, que se corresponde con el caso en el que el autovalor es positivo, luego el equilibrio sería repulsivo. El segundo caso es cuando la diferencia $a-b<0$, es decir, $a<b$ o de la ecuación (11), $p>1$, que se corresponde con el caso en el que el autovalor es negativo, luego el equilibrio sería atractivo.

El cálculo sobre el/los otro(s) valor(es) de equilibrio se hace complicado, aunque no imposible, por lo que se reconsidera una forma que pueda ser más simple para la obtención de la estabilidad del/los punto(s) de equilibrio restantes.

Nótese que graficar simultáneamente la ecuación $x=0$ y la ecuación (12) permite esa opción, pero adicionalmente entrega un valor agregado: el diagrama de bifurcaciones del sistema (Figura 5).

El diagrama de bifurcaciones es un diagrama en el que se grafican parámetros vs variables de estado, con la salvedad de que al variar el parámetro seleccionado, deben ocurrir cambios en el número de puntos de equilibrio o en la estabilidad de estos últimos, luego no puede ser cualquier parámetro. El parámetro que por su variación conduce a cambios en la estructura de las órbitas del sistema se denomina parámetro de bifurcación. Para el ejemplo que se ha abordado, el parámetro de bifurcación es el parámetro $p=b / a$.

Entonces, como se puede observar (Figura 5), se tiene que para $0<p<1$, se cuenta con dos puntos de equilibrio para cada línea fase -recta vertical-, los cuales son el cero y un valor positivo de $x$, donde el cero es repulsivo y el valor positivo de $x$ es atractivo. Este resultado se desprende de los casos definidos a partir de la ecuación (20).

Al aumentar el valor del parámetro $p$, de modo que $1<p<p_{e^{\prime}}$ se observa que cada línea fase ahora cuenta con 
tres puntos de equilibrio, dos atractivos y uno repulsivo, lo cual es denominado biestabilidad.

Figura 5. Diagrama de bifurcaciones

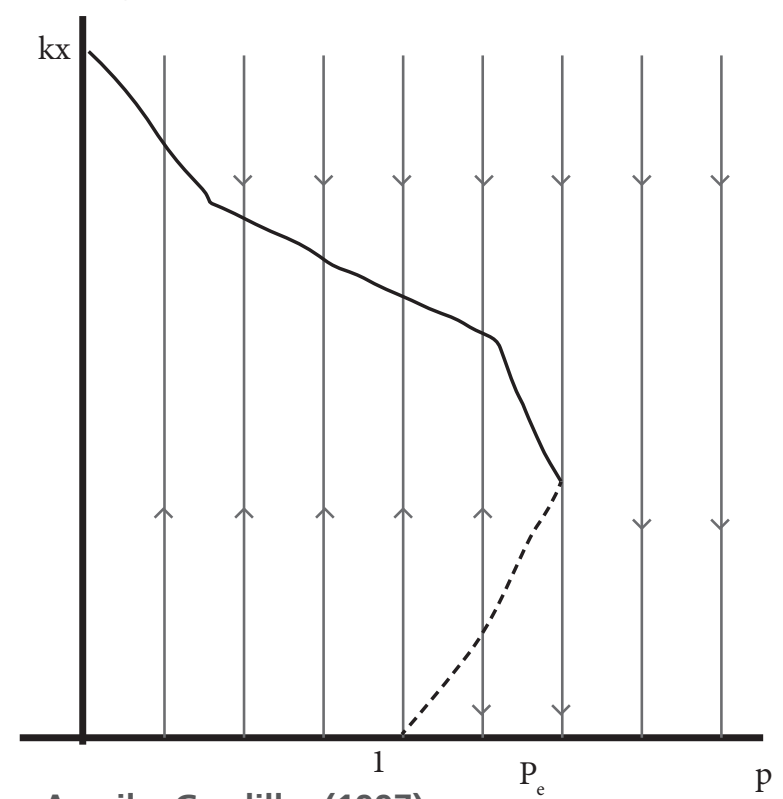

Fuente. Aracil y Gordillo, (1997).

Finalmente, para $p>p_{e^{\prime}}$ las líneas de fase solo cuentan con un único punto de equilibrio atractivo.

Ahora, es posible saber qué sucedía en el sistema para las simulaciones presentadas con anterioridad (Figura 4). Lo que ocurría era que el sistema tenía su parámetro de bifurcación p en la zona de biestabilidad, por lo que se veía decrecer para unos valores y crecer para otros. Pero adicionalmente, ahora es posible ver que el sistema tenía mayor riqueza, porque puede pasar de dos equilibrios a tres y a uno con sus correspondientes cambios de estabilidad. Esto es lo que se señala como cambios de escenarios prospectivos, con lo que la toma de decisiones es completamente asertiva sobre los puntos de apalancamiento del sistema y el reconocimiento de sus resultados. 


\section{Mapas de todos los escenarios posibles y la toma de decisiones}

Como se vio en la sección anterior, existen unos diagramas que permiten el reconocimiento de los cambios cualitativos de la estructura de las trayectorias de los sistemas denominados diagramas de bifurcación.

El principal aporte de este documento se encuentra en la interpretación de los diagramas de bifurcación como mapas de todos los escenarios posibles. Es decir, dichos diagramas son herramientas que permiten explicar el comportamiento prospectivo de los sistemas sobre los que actuamos para el reconocimiento cualitativo de los resultados que se obtendrán sobre los sistemas cuando se intervienen. Los escenarios futuros que se definen de esta interpretación se denominarán escenarios prospectivos.

A partir de esta interpretación y volviendo sobre el problema presentado en la sección anterior, se puede realizar el siguiente análisis:

- Para valores de p entre cero y uno, el sistema tiende a crecer hacia un valor positivo de $x$, que como se recordará, es el número de empresas. Esta tendencia creciente se interpreta como el primer escenario prospectivo del sistema.

- Para valores de $\mathrm{p}$ entre uno y un valor denominado $p_{e^{\prime}}$ el sistema se hace biestable, lo cual significa que se debe estar por encima del valor inestable para tender a un estado de equilibrio positivo en el que exista un número importante de empresas o de lo contrario, el número de empresas tenderá a desaparecer. Esta intrincada tendencia se interpretará como un segundo escenario prospectivo del sistema. 
- Finalmente, si los valores de $p$ están por encima de $p_{e^{\prime}}$ el número de empresas tenderá a desaparecer, con lo cual se define un tercer escenario prospectivo para el sistema.

De este modo, partiendo del diagrama de bifurcaciones, fue posible establecer tres escenarios prospectivos del sistema: el conveniente, el intrincado y el inconveniente. En este punto, la toma de decisiones se convierte en un ejercicio de elección sobre la base de una comprensión más robusta del sistema. Ahora es posible ver con claridad las claras consecuencias en el tiempo de lo que se puede decidir.

Para el caso del problema que se ha considerado en este documento, el propósito era establecer la mayor cantidad de empresas en la zona industrial, para lo cual, las decisiones definían tres escenarios, siendo el más conveniente aquel en el que el parámetro de bifurcación $p$ fuera tan pequeño como fuera posible; pero el parámetro $p$ se había definido como el cociente entre $b$ y $a$, que corresponden con la vida media empresarial $V M E$ y la tasa normal de creación de empresas TNCE, luego:

$$
p \longrightarrow 0
$$

Es lo mismo que:

$$
p=\frac{b}{a}=\frac{1}{T N E}=\frac{1}{T N C E \cdot V M E} \rightarrow 0
$$

De donde concluimos finalmente que lo que se busca sobre el sistema es:

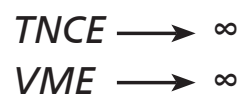

Es decir, que la tasa normal de creación de empresas y/o la vida media empresarial sean tan grandes como sea posible. De ahí, que cualquier decisión que se tome debe satisfacer que al menos una de las dos condiciones se cumpla. 


\section{Conclusiones}

Se ha estudiado un problema en el que se debe decidir, a partir de la simulación, las políticas que se deben establecer para ocupar un área que se estableció para el desarrollo industrial de una región. En ese orden de ideas, la realización de ensayos que desde el error digan qué hacer, podría resultar costoso en tiempo, en dinero y en prestigio. Entonces, tener una herramienta como el diagrama de bifurcaciones es de mucha utilidad.

El diagrama de bifurcaciones es, en sí mismo, un mapa de todas las posibilidades dinámicas del sistema y, por lo tanto, un instrumento que nos permitirá proponer escenarios prospectivos y las acciones que se deben tomar para que los parámetros del sistema que determinan ese comportamiento dinámico, conduzcan al escenario deseado.

Es importante señalar que, aunque los diagramas de bifurcación son herramientas magníficas para la toma de decisiones, su generación es, de acuerdo al sistema, un ejercicio que puede resultar complicado y, por lo tanto, con las técnicas actuales, no siempre pueden generarse a la velocidad que demanda el tomador de decisión. 


\section{Referencias bibliográficas}

Arnold, V. (1973). Ordinary diferential equations. Cambridge, MA: MIT Press.

Cohen, D. \& Henle, J. (2005). Calculus, the language of change. Sudbury, Massachusetts: Jones and Bartlett Publishers.

Di Bernardo, M., Budd, C.J., Champneys, A.R. \& Kowalczyk, P. (2008). Piecewise-smooth dynamical systems, theory and applications. New York: Springer-Verlag.

Guckenheimer, J. \& Holmes, P. (1983). Nonlinear oscillations, dynamical systems, and bifurcations of vector fields. New York: Springer-Verlag.

Hirsch, M. \& Smale, S. (1974). Diferential equations, dynamical systems, and linear algebra. New York: Academic Press

Kuznetsov, Y.A. (1998). Elements of applied bifurcation theory. New York: Springer-Verlag. Second Edition.

Lynch, S. (2004). Dynamical systems with applications using matlab. Boston: Birkhauser.

Wiggins, S. (1990). Introduction to applied nonlinear dynamical systems and chaos. New York: Springer-Verlag. 